\title{
An application of recurrent neural networks to discriminative keyword spotting
}

\author{
Santiago Fernández ${ }^{1}$, Alex Graves ${ }^{1}$, and Jürgen Schmidhuber ${ }^{1,2}$ \\ 1 IDSIA, Galleria 2, 6928 Manno-Lugano, Switzerland \\ 2 TU Munich, Boltzmannstr. 3, 85748 Garching, Munich, Germany
}

\begin{abstract}
Keyword spotting is a detection task consisting in discovering the presence of specific spoken words in unconstrained speech. The majority of keyword spotting systems are based on generative hidden Markov models and lack discriminative capabilities. However, discriminative keyword spotting systems are based on the estimation of a posteriori probabilities at the frame-level, hence they make use of information from short time spans. This paper presents a discriminative keyword spotting system based on recurrent neural networks only, that uses information from long time spans to estimate keyword probabilities. In a keyword spotting task in a large database of unconstrained speech where an HMM-based speech recogniser achieves a word accuracy of only $65 \%$, the system achieved a keyword spotting accuracy of $84.5 \%$.
\end{abstract}

\section{Introduction}

Keyword spotting is a detection task consisting in discovering the presence of specific spoken words in (typically unconstrained) speech signals. Applications of this technology include audio indexing, detection of command words in interactive environments and spoken password verification. In general, the technology is applied to domains in which a full speech recogniser is cumbersome to develop and unnecessary, partly because less than perfect detection rates are still very satisfactory.

Nonetheless, the same mathematical framework used in the majority of speech recognisers forms the basis for keyword spotting systems too. The typical keyword spotting system consists of a set of hidden Markov models (HMM), one for each keyword plus one or more filler models [1]. The filler models characterize non-keyword events in the speech signal, such as other spoken words, background noises and silence. The approach is generative and finds the most likely sequence of models that produces the observations. The output of the system is post-processed before deciding on the presence or absence of keywords in the utterance [2]. First, the confidence on the predictions is measured. This is typically done by computing the ratio of likelihoods between keyword model hypotheses and filler model hypotheses. Finally, a threshold level is applied to the confidence measure in order to achieve a compromise between the number of true and false positives predicted by the system. 
In general, a discriminative approach to keyword spotting is more suitable, as it allows discriminating between keyword and non-keyword events, and also among similar keywords. Besides this, posterior probabilities can directly be used as an effective confidence measure. Recently, discriminative keyword spotting systems have been explored in various papers. The most extended approach [3$5]$ consists in using artificial neural networks (ANN) in the framework of hybrid ANN/HMM systems [6,7]. In this setup, frame-level posteriors are estimated with, typically, a multi-layer perceptron (MLP). These local posterior estimates are accumulated over the duration of a segment and the result normalised by the length of the segment. Another approach to discriminative keyword spotting uses kernel machines and large margin classifiers with a set of feature functions consistent with hybrid ANN/HMM models [8].

The approach presented in this paper attempts to model full keywords in the sequence data stream, while previous discriminative keyword spotting systems are based on sub-word units (phones). It is based on recurrent neural networks (RNN) and the connectionist temporal classification (CTC) algorithm. The algorithm $[9,10]$ is a general framework for labelling unsegmented sequential data with artificial neural networks. The proposed system uses information over long time spans for providing non-local estimates of the a posteriori probability of the presence of either a keyword or non-keyword events in the speech signal.

There is a plethora of different keyword spotting systems, often tailored to meet the requirements of particular tasks, and little consensus to define benchmark tasks [11]. We have opted to tackle a realistic keyword spotting task in a large database of spontaneous and unconstrained speech where an HMM-based speech recogniser achieves a word accuracy of only $65 \%$.

The remainder of the paper is structured as follows. Section 2 provides a description of the system. Keyword spotting experiments are presented in section 3 and the results are shown in section 3.3. Section 4 offers a discussion on differences between previous approaches and the one presented in this paper, and gives directions for future work. Final conclusions are given in section 5 .

\section{Method}

\subsection{Outline}

The network architecture selected for the keyword spotting task is the bidirectional long short-term memory recurrent neural network (BLSTM), which has shown good performance in a series of speech tasks $[12,13]$. The network is trained with the connectionist temporal classification (CTC) algorithm $[9,10]$.

The input to the recurrent network is the data sequence of a speech utterance. The network has a soft-max output layer with as many output units as keywords have to be detected, plus one output unit associated with non-keyword events. The target for the training algorithm is a list (which may be empty) of keywords in the order in which they appear in the input speech utterance. No further constraints are applied to the system, e.g. the segmentation of the speech signal 


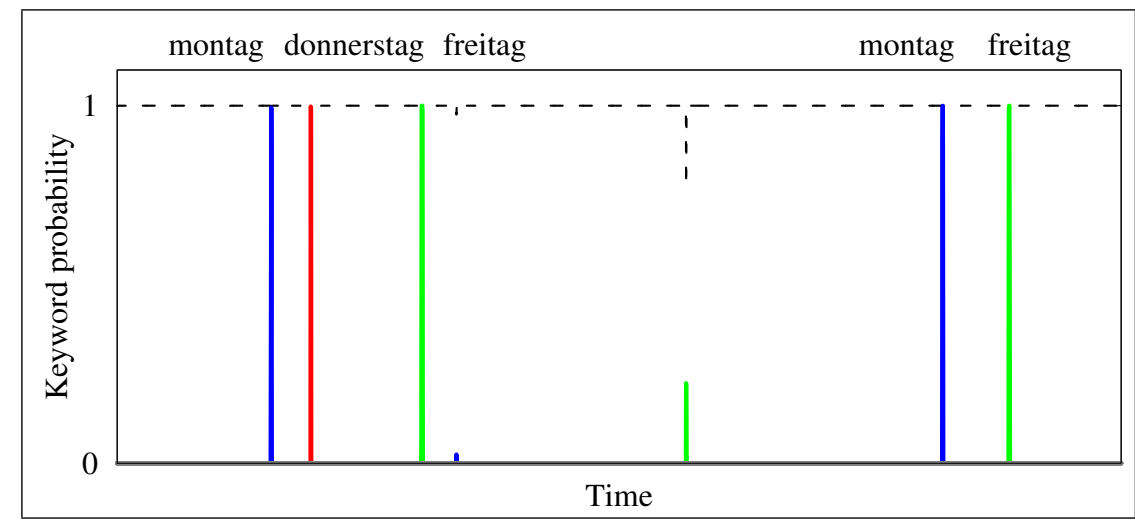

Fig. 1. Discriminant keyword spotting with BLSTM and CTC. When a keyword is detected the ANN produces a spike-like output with higher probability than the nonkeyword output (dashed line).

is not required and various pronunciation variants of the same keyword can appear in the data set.

Once the network has been trained, its output will, typically, consist in a series of spikes corresponding to keyword events that have been detected in the input, and separated by long periods of activation of the non-keyword output unit (see figure 1). The activation of every output unit at every time step corresponds to the probability of detecting a particular keyword (or non-keyword event) at a particular time step.

\subsection{BLSTM}

The long short-term memory (LSTM) $[14,15]$ is an RNN architecture designed to deal with long time-dependencies and addresses the problem of the backpropagated error either blowing up or decaying exponentially for long time lags in conventional RNNs. The hidden layer of LSTM networks consists of a set of recurrently connected blocks containing one or more memory cells and three multiplicative units (the input, output and forget gates), which allow writing, reading or resetting the information in the memory cell.

Bi-directional RNNs [16] address in an elegant way the need for delayed decisions in some sequential tasks such as speech processing. Data sequences are presented forwards and backwards to two separate recurrent networks connected to the same output layer. Therefore, for every point in a given sequence, the network has complete sequential information about (some of) the points before and after it. An implementation of the bi-directional LSTM (BLSTM) can be found in [12]. 


\subsection{CTC}

Connectionist temporal classification (CTC) $[9,10]$ is an algorithm to label unsegmented sequential data with RNNs. The basic idea behind the algorithm is to interpret the network outputs as a probability distribution over all possible label sequences, conditioned on the input data sequence. Given this distribution, an objective function can be derived that directly maximises the probabilities of the correct labellings. Since the objective function is differentiable, the network can be trained with standard back-propagation through time. The algorithm requires that the network outputs at different times are conditionally independent given the internal state of the network. This requirement is met as long as there are no feedback connections from the output layer to itself or to the network.

\section{Experiments}

\subsection{Material}

The experiments were carried out on a set of dialogues in German from the Verbmobil database. The dialogues deal with the scheduling of date appointments for meetings. The database consist in spontaneous and unconstrained speech, with long silences and noises. The results provided along with the database for a baseline automatic speech recogniser give an approximate idea of the difficulty of processing this database: the HMM-based speech recogniser achieved a word accuracy of $65 \%^{3}$.

The material used in the experiments corresponds to version 2.3 (March 2004) of the Verbmobil database (except for CD-ROM number 53.1 of the training set which was not available). It includes separate training, validation and test sets. The database is speaker independent. Speakers were distributed equally across sexes in all sets and every speaker appears in only one of the sets. The training set includes 748 speakers and 23975 dialogue turns for a total of 45.6 hours of speech. The validation set includes 48 speakers and 1222 dialogue turns for a total of 2.9 hours of speech. The test set includes 46 speakers and 1223 dialogue turns for a total of 2.5 hours of speech.

Due to the nature of the dialogues included in the database, we decided to use dates and places as keywords for the detection task. This ensures a relatively good coverage of keywords in the training, validation and test data sets. The twelve keywords chosen were:

april, august, donnerstag, februar, frankfurt, freitag, hannover, januar, juli, juni, mittwoch, montag

Note that the database include various pronunciation variants of some of these keywords (e.g. "montag" can end either with a /g/ or with a /k/). In addition,

\footnotetext{
${ }^{3}$ We tried training an HMM-based keyword spotting system, with as few constraints as those required by our system, for this keyword spotting task but it did not converge.
} 
several keywords appear as sub-words, e.g. in plural form such as "montags" or as part of another word such as "ostermontag" (Easter Monday).

Therefore, the orthographic transcription provided along with the database was examined for the presence in every utterance of one or various of the twelve keywords selected. Once a keyword was found, the begin and end times for the keyword were saved for evaluating the performance of the keyword spotting system at a later stage. These times were provided along with the database and correspond to the segmentation given by an automatic speech recognition system.

This procedure gave a total of 10469 keywords on the training set with an average of $1.7 \%$ keywords per non-empty utterance $(73.6 \%$ of the utterances did not have any keyword); 663 keywords on the validation set with an average of $1.7 \%$ keywords per non-empty utterance $(68.7 \%$ of the utterances did not have any keyword); and 620 keywords on the test set with an average of $1.8 \%$ keywords per non-empty utterance $(71.1 \%$ of the utterances did not have any keyword).

The list of keywords (which may be empty) in the order in which they appear in every utterance, forms the target sequence for the network's training algorithm.

Finally, the speech data was characterised as a sequence of vectors of thirty nine coefficients, consisting of twelve Mel-frequency cepstral coefficients (MFCC) plus energy and first and second order derivatives of these magnitudes. The coefficients were computed every $10 \mathrm{~ms}$ over $25 \mathrm{~ms}$-long windows. First, a Hamming window was applied, secondly, a mel-frequency filter bank of 26 channels was computed and, finally, MFCC coefficients were calculated with a 0.97 preemphasis coefficient. The input data was normalised to have zero mean and standard deviation one.

\subsection{Setup}

The CTC-BLSTM network was trained with the input sequences and keyword targets from section 3.1. The network has, therefore, 39 input units and 13 units in the output soft-max layer. Both the forward and backward hidden recurrent layers consist of 128 LSTM memory blocks with one memory cell per block, peephole connections and forget gates. The input and output cell activation functions are a hyperbolic tangent. The gates use a logistic sigmoid function in the range $[0,1]$. The input layer is fully connected to the hidden layer and the hidden layer is fully connected to itself and to the output layer. The total number of weights in the network is 176141.

Training was carried out with a learning rate of $10^{-4}$ and a momentum coefficient of 0.9 . Weights were initialised randomly in the range $[-0.1,0.1]$. In addition, Gaussian noise with a standard deviation of 0.5 was added to the inputs during training to improve generalization. The network was tested every five epochs on the validation set and training finished once the error on the validation set stopped decreasing. 


\subsection{Results}

The typical sequence of outputs for a trained network can be seen in figure 1. It consists in a sequence of spikes associated with detected keywords and separated by long periods during which the non-keyword output unit is the most active. The CTC algorithm allows the network to keep any output active for as long as necessary or as little as one time step only. To evaluate the results, at every time step the output with the highest activation is chosen. If an output is the most active for a period of time, we have chosen the highest activation during that period as the probability of detecting the keyword, and the time at which the highest activation occurs as the location of the detected keyword. It is easy to avoid this step, though, by a simple modification to the CTC algorithm that constraints keyword outputs to last for one time step only.

Spikes whose identity match a keyword in the speech signal and that appear within the boundaries of the keyword in the speech signal are counted as true positives, unless more than one spike is output in that period, in which case only one of them counts as a true positive and the rest count as false positives. Those spikes that appear out of the boundaries of the keyword in the speech signal are considered false positives. The accuracy of the system is given by the number of true positives minus the number of false positives divided by the number of keywords in the data set.

Four networks were trained on the same task with different initial random weights. Average accuracy over four runs was $84.5 \%$ with a standard error of $1.2 \%$. The average probability of true positives was 0.98 with a standard error of 0.004 . The average probability of false positives was 0.80 with an standard error of 0.01. As expected, given the discriminative nature of the algorithm, setting a posteriori a threshold level in between this probability levels did not increase performance significantly ( $84.8 \%$ accuracy, with an standard error of $1.2 \%$ ). Table 1 shows the number of true and false positives by keyword for the network with the best performance. As shown in the table, the system discriminates almost perfectly between similar keywords such as "juni" and "juli".

As of now, the CTC training algorithm does not constrain the spikes to appear within the begin and end times of the speech pattern. The algorithm trains the network to detect the correct sequence of keywords and in the right order for any utterance in the training set. Probably this makes training slower, but it seems that it is not necessary to add this constraint to the training algorithm in order to achieve good performance: when the results are evaluated without using the segmentation, the average accuracy over four runs is $86.1 \%$ with a standard error of $0.7 \%$. Of those keyword candidates appearing outside the boundaries in the speech signal, only one was more than $0.5 \mathrm{~s}$ outside the reference segment. This level of precision can be enough for tasks where high detection accuracy is more important.

Finally, figure 2 shows, for a successful detection, the dependency of the network output at the time when the keyword is detected with respect to the inputs at some previous and following time steps. As can be seen, the probability of detecting the keyword depends on the inputs over a long time span 
Table 1. Results by keyword for the ANN with the best performance. Accuracy is the number of hits minus false positives divided by the actual number of keywords in the test set. Average accuracy over four runs is $84.5 \%$ with a standard error of $1.2 \%$.

\begin{tabular}{|r|c|c|c|c|}
\hline Keyword & \# Hits & \# FPs & \# Actual & \% Accuracy \\
\hline \hline april & 27 & 2 & 32 & 78.12 \\
august & 29 & 1 & 34 & 82.35 \\
donnerstag & 55 & 6 & 56 & 87.50 \\
februar & 55 & 1 & 60 & 90.00 \\
frankfurt & 18 & 0 & 25 & 72.00 \\
freitag & 40 & 4 & 45 & 80.00 \\
hannover & 76 & 5 & 86 & 82.56 \\
januar & 35 & 4 & 38 & 81.58 \\
juli & 53 & 1 & 56 & 92.86 \\
juni & 63 & 2 & 66 & 92.42 \\
mittwoch & 36 & 1 & 39 & 89.74 \\
montag & 79 & 3 & 83 & 91.57 \\
Overall & 566 & 30 & 620 & 86.45 \\
\hline
\end{tabular}

(of about one second, or a hundred network time steps) and, for the example shown, the dependency decreases towards the end of the keyword, which is the least discriminative part of it.

\section{Discussion and Future Work}

The main difference between our approach to discriminative keyword spotting and other discriminative systems is the capability of computing non-local posteriors for the keywords, i.e. the system uses information over long time spans to compute the probability of a keyword appearing in the speech signal. In addition to this, the a posteriori estimation of a threshold level to balance true and false positives is not required.

The algorithm makes very few assumptions about the domain, which facilitates the development of systems for detection tasks. Nonetheless, if very high precision in the location of spots is required, the system might benefit from adding to the algorithm the constraint that keywords must be detected within the boundaries established by the reference segmentation. However, not having this constraint greatly simplifies the preparation of training data.

To make the system scale, one solution consists in implementing smaller networks. For example, one for each keyword, or one for each set of similar keywords among which the system must discriminate. New networks can be added to the system at any time. Under the assumptions that, for different networks, the training data has similar characteristics and that the non-keyword output unit is associated to a model of similar characteristics, which is reasonable, the a posteriori probabilities given by different networks can be compared and used to determine the presence or absence of keywords in the input data stream. 


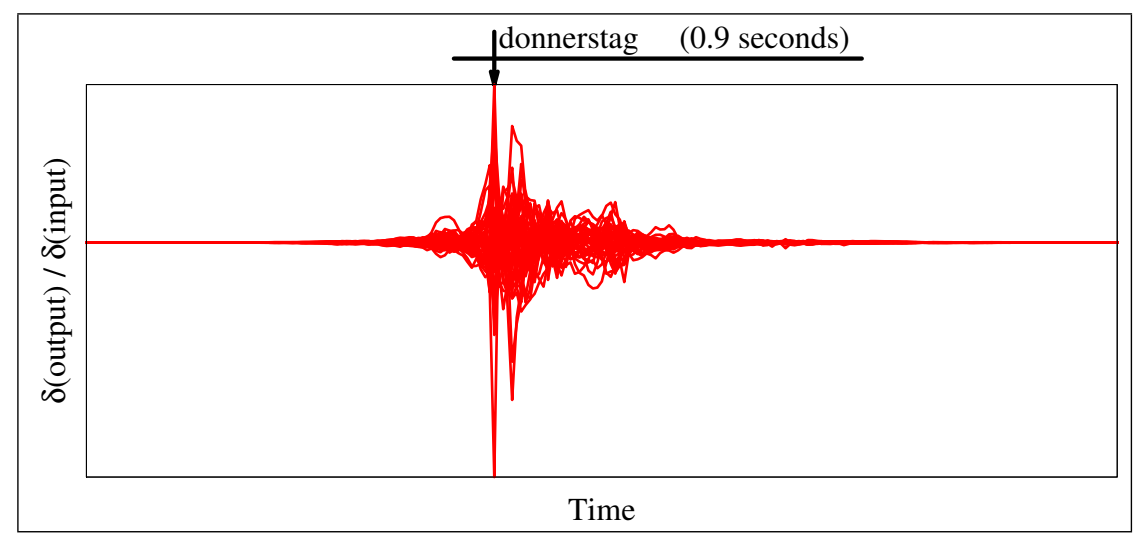

Fig. 2. Example of network outputs dependency with respect to the inputs. The figure shows the dependency of the output unit associated with keyword "donnerstag" at the time step when this keyword is detected in figure 1, with respect to the network inputs at various time steps around the detection point (indicated by an arrow at the top of the figure). In addition, the extent $(0.9 \mathrm{~s})$ and location of the keyword in the speech signal is shown at the top of the figure. As can be seen, the probability of detecting the keyword depends on the inputs over a long time span, and decreases towards the end of the keyword which is the least discriminative part of it: "tag" (day).

Another option to improve scalability consists in detecting sub-word units in the signal and use these outputs as inputs for one of the existing systems that builds keywords from sub-word units. The detection of sub-word units with the system proposed in this paper will benefit from the same features and ease of use described in this paper (see [9] for results on phoneme recognition with CTC-BLSTM). Besides this, it is possible to feed the sub-word level predictions to another CTC-BLSTM network with keyword-level outputs [10].

The choice of sub-word or word models depends on the task. In general, sub-word units are more practical for audio indexing tasks because the system must respond to searches for unknown keywords. In tasks such as detection of command words and spoken password verification, it is likely that the vocabulary is either fixed or it does not change so often that it is not possible to update the system. Also, for safety reasons, it is in these cases in which the benefits of a word-level discriminative keyword spotting system are most desirable. Namely, the capability of decreasing confusion among similar keywords by discriminating among them and between non-keyword and keyword events.

\section{Conclusion}

We have presented a word-level discriminative keyword spotting system that is easy to develop and use, fast and accurate. The probability of detecting a keyword is computed using information from long time spans. Those probabilities can be read directly from the outputs of a recurrent neural network. An a 
posteriori estimate of an acceptance threshold level is not required. Finally, the system makes few assumptions about the domain: only the input speech signal and a list of keywords (which may be empty) in the order in which they occur in the signal are necessary to train the system. The algorithm is general and can be used for any task requiring the detection of patterns in sequence data. In a keyword spotting task in a large database of unconstrained speech where an HMM-based speech recogniser achieves a word accuracy of only $65 \%$, the system achieved a keyword spotting accuracy of $84.5 \%$.

\section{Acknowledgments}

This research was funded by SNF grant 200021-111968/1.

\section{References}

1. Wilpon, J.G., Rabiner, L.R., Lee, C., Goldman, E.R.: Automatic recognition of keywords in unconstrained speech using hidden Markov models. IEEE Transactions on Acoustics, Speech and Audio Processing 38(11) (1990) 1870-1878

2. Benítez, M.C., Rubio, A., García, P., de la Torre, A.: Different confidence measures for word verification in speech recognition. Speech Communication 32 (2000) 79-94

3. Silaghi, M.C., Bourlard, H.: Iterative posterior-based keyword spotting without filler models. In: Proceedings of IEEE Automatic Speech Recognition and Understanding Workshop, Keystone - Colorado (U.S.A.) (1999)

4. Silaghi, M.: Spotting subsequences matching an HMM using the average observation probability criteria with application to keyword spotting. In: Proceedings of the 20th National Conference on Artificial Intelligence and the 17th Conference on Innovative Applications of Artificial Intelligence, Pittsburgh - Pennsylvania (U.S.A.) (2005) 1118-1123

5. Ketabdar, H., Vepa, J., Bengio, S., Bourlard, H.: Posterior based keyword spotting with a priori thresholds. In: Proceedings of the 9th International Conference on Spoken Language Processing, Pittsburgh - Pennsylvania (U.S.A.) (2006)

6. Robinson, A.J.: An application of recurrent nets to phone probability estimation. IEEE Transactions on Neural Networks 5(2) (1994) 298-305

7. Bourlard, H.A., Morgan, N.: Connectionist speech recognition: a hybrid approach. Kluwer Academic Publishers (1994)

8. Keshet, J., Grangier, D., Bengio, S.: Discriminative keyword spotting. In: Workshop on Non-Linear Speech Processing (NOLISP), Paris (France) (2007)

9. Graves, A., Fernández, S., Gomez, F., Schmidhuber, J.: Connectionist temporal classification: labelling unsegmented sequence data with recurrent neural nets. In: Proceedings of the 23rd International Conference on Machine Learning, Pittsburgh - Pennsylvania (U.S.A.) (2006)

10. Fernández, S., Graves, A., Schmidhuber, J.: Sequence labelling in structured domains with hierarchical recurrent neural networks. In: Proceedings of the 20th International Joint Conference on Artificial Intelligence, Hyderabad (India) (2007)

11. Silaghi, M.C., Vargiya, R.: A new evaluation criteria for keyword spotting techniques and a new algorithm. In: Proceedings of InterSpeech. (2005) 
12. Graves, A., Schmidhuber, J.: Framewise phoneme classification with bidirectional LSTM and other neural network architectures. Neural Networks 18(5-6) (2005) 602-610

13. Graves, A., Fernández, S., Schmidhuber, J.: Bidirectional LSTM networks for improved phoneme classification and recognition. In: Proceedings of the 15th International Conference on Artificial Neural Networks, Warsaw (Poland) (2005)

14. Hochreiter, S., Schmidhuber, J.: Long short-term memory. Neural Computation 9(8) (1997) 1735-1780

15. Gers, F., Schraudolph, N., Schmidhuber, J.: Learning precise timing with LSTM recurrent networks. Journal of Machine Learning Research 3 (2002) 115-143

16. Schuster, M., Paliwal, K.K.: Bidirectional recurrent neural networks. IEEE Transactions on Signal Processing 45 (1997) 2673-2681 